Revue d'histoire de l'enfance « irrégulière »

Le Temps de l'histoire

6 | 2004

Les sciences du psychisme et l'enfance « irrégulière »

\title{
Bertrand Ravon L'« échec scolaire ». Histoire d'un problème public
}

Paris, In Press éditions, 2000, 380 p.

Dominique Dessertine

\section{(2) OpenEdition}

\section{Journals}

Édition électronique

URL : http://journals.openedition.org/rhei/119

DOI : $10.4000 /$ rhei. 119

ISBN : 978-2-7535-1644-1

ISSN : 1777-540X

Éditeur

Presses universitaires de Rennes

Édition imprimée

Date de publication : 15 novembre 2004

Pagination : 308-310

ISSN : 1287-2431

\section{Référence électronique}

Dominique Dessertine, «Bertrand Ravon L'« échec scolaire ». Histoire d'un problème public », Revue d'histoire de l'enfance « irrégulière » [En ligne], 6 | 2004, mis en ligne le 15 novembre 2004, consulté le 03 décembre 2020. URL : http://journals.openedition.org/rhei/119; DOl : https://doi.org/10.4000/rhei. 119 


\section{Bertrand Ravon \\ L'«échec scolaire ". Histoire d'un problème public \\ Paris, In Press éditions, 2000, 380 p.}

Cet ouvrage, issu d'une thèse de sociologie, essaie de repérer l'émergence d'un problème, nommé à la Libération, devenu politique au début des années soixante, en le liant étroitement à l'émergence des savoirs qui en ont fourni les grilles de lecture. Si les sociologues jouent un rôle important dans son approche au cours du dernier tiers du Xxème siècle, les psychologues des débuts de la Troisième République, époque de la scolarisation obligatoire de tous, et de grands pédagogues ont posé les premiers jalons de ce qu'ils nomment alors le " retard scolaire ». Les médecins, qui apparaissent dans le champ au cours de l'entre-deux-guerres et consolident leur influence dans les années cinquante, sont aussi pris en compte. Tous les spécialistes de la pédagogie qui se sont penchés sur les réalités scolaires apparaissent dans cet ouvrage. Aussi est-il précieux tout autant à ceux qui s'intéressent spécifiquement au problème étudié qu’à tous ceux qui seraient désireux de disposer d'une histoire des hommes, des réseaux (parisiens essentiellement) qui se sont mêlés de pédagogie. L'auteur fournit de rapides biographies de tous les grands personnages impliqués dans cette histoire, les resituent dans leur cursus familial, social, professionnel et intellectuel, pour bien rappeler que les idées de chacun émanent certes de son intelligence mais sont aussi portées par des courants, des réseaux, des réussites ou des échecs professionnels. Cette histoire intellectuelle ne tombe pas dans l'écueil d'une histoire désincarnée et désocialisée. Comme l'écrit dans la préface Dominique Glasman, les personnages qui ont porté cette histoire sont impliqués personnellement : «l'échec scolaire » les concerne au sens le plus fort du terme.

Tout commence donc avec la Troisième République et la psychopédagogie autour de Binet, qui contribue à l'émergence du " retard scolaire ", qui trouvera son remède (officiel) avec la loi de 1909 sur les classes de perfectionnement, très largement restées lettre morte ou détournées de leur but initial. L'auteur montre bien l'évolution de Binet, de la psychologie vers

Dominique Dessertine / p. 308 à 310 
la sociologie. Tranchant de tous les débats en homme de science, et auteur de la première étude française portant sur des élèves d'école primaire, il est peu à peu conduit, dans ses enquêtes multipliées, à repérer la misère physiologique de beaucoup d'enfants, à l'intégrer dans la grille de lecture du retard scolaire. Mais la sociologie ne pénètre pas pour autant la Société libre pour l'étude psychologique de l'enfant, fondée par Ferdinand Buisson et dont Binet est un des maillons essentiels, même si elle contribue à rendre le débat public. Car Durkheim se méfie de la psychologie et sa sociologie s'interdit de s'accomplir dans la pratique. Le débat sur la fonction sociale de l'école porte alors implicitement sur les risques que de trop nombreux diplômés issus du bas de l'échelle feraient courir à la société. Que deviendraient-ils face à un monde étranger à leurs valeurs d'origine qui les refuserait ? Quels troubles feraient naittre ces exclus, qui seraient de fait écartés au profit des détenteurs traditionnels des postes et des pouvoirs ? Le retard scolaire est donc un problème tout à fait secondaire socialement.

Le débat sur l'école unique monopolise l'entre-deux-guerres. Une même nation à la bataille de la Marne, une même nation à l'école. Avec le Cartel des gauches, Buisson préside la Commission sur l'école unique. En 1928, Herriot amorce la gratuité des collèges, effective en 1933. On sait que l'école unique sera définitivement (?) instituée par la réforme Haby de 1975. Le plan Langevin-Wallon, partant du principe que la profession sépare et que la culture rapproche, pose de façon nouvelle la question de l'inégalité des enfants devant l'acquisition des savoirs. Tant que la république s'était contentée de généraliser l'accès de tous au primaire, le problème restait circonscrit au premier niveau de l'enseignement. L'enseignement secondaire n'accueillait que ceux qui avaient donné antérieurement des gages de leurs aptitudes. Désormais les problèmes se posent différemment.

Le lecteur pourra suivre aussi, dans ce livre, le processus de l'émergence institutionnelle de la psychologie en milieu scolaire, la création par Henri Wallon, ministre de l'Éducation nationale en 1945, du premier centre de psychologie scolaire à Grenoble et la mise en place des premiers postes de psychologues scolaires. Wallon édite aussi la revue Enfance, qui publie sous la plume de Snyders les premières critiques du Binet-Simon-Terman. Le 
lecteur abordera aussi l'arrivée de la psychanalyse dans ce milieu, avec la fondation, l'année suivante, par Georges Mauco, psychanalyste nonmédecin, du centre psychopédagogique (CPP) du 13ème arrondissement de Paris. Les CMPP, créés en 1956, prennent position contre les pratiques médico-psychologiques de la neuropsychiatrie infantile.

Ainsi, à l'aide de ce livre très riche, dont on n'a repris ici que quelques thèmes forts, le lecteur pourra retrouver les grands débats abordés dans ce numéro du Temps de l'bistoire. L'échec scolaire, comme la "rééducation" des délinquants, a été l'objet des mêmes débats, orchestrés par les mêmes spécialistes et portés par les mêmes enjeux sociaux. En décortiquant la naissance d'un "concept", Bernard Ravon appelle à conserver un esprit d'analyse face à une notion qui pourrait sembler "objective".

Dominique Dessertine 